\title{
Ammonium-functionalized poly(arylene ether)s as anion-exchange membranes
}

\author{
Junpei Miyake $^{1,2}$, Masahiro Watanabe ${ }^{1}$ and Kenji Miyatake Me,2,3 $^{1,2}$ \\ The recent progress of our research on anion-exchange membranes (AEMs) for alkaline fuel cell applications is reviewed. The \\ anion conductivity and the mechanical, thermal and chemical stability of AEMs are insufficient. To address these issues, we \\ have designed and synthesized a novel series of poly(arylene ether)-based AEMs with quaternized ammonium groups. First, the \\ effect of the sequence of the polymer main chain (random or block) on the AEM properties, especially the anion conductivity, is \\ discussed. We emphasize that fluorenyl groups serve effectively as scaffolds for the ammonium groups. We then discuss the \\ alkaline stability of both the polymer backbone and the quaternized ammonium groups. Partial fluorination improves the \\ alkaline stability of the polymer main chains. Among the several ammonium groups investigated, we propose that pyridinium \\ groups are seemingly more stable than the typical aliphatic ammonium (for example, trimethylammonium) groups. The results \\ imply that aromatic AEMs are potentially applicable to alkaline fuel cells that use hydrogen or hydrazine as a fuel. \\ Polymer Journal (2014) 46, 656-663; doi:10.1038/pj.2014.56; published online 16 July 2014
}

\section{INTRODUCTION}

Anion-exchange membranes (AEMs) and related cationic polymers have a variety of applications in electrochemical devices, including fuel cells (FCs), metal-air batteries, water electrolyzers, redox flow batteries, electrodialyzers and sensors. ${ }^{1-11}$ Among these applications, anion-exchange membrane fuel cells (AEMFCs) have recently attracted considerable attention because of their advantages over proton-exchange membrane (PEM) fuel cells. ${ }^{12,13}$ These advantages include their potential use of non-precious metals as electrocatalysts and better oxygen-reduction reaction kinetics. Their ability to use non-precious metals as electrocatalysts has been demonstrated in an AEMFC system, ${ }^{14}$ in which chromium-decorated nickel and silver were used as the anode and cathode, respectively. The critical issues associated with AEMFCs are insufficient ion conductivity and the instability of the existing AEMs at high temperatures and/or under alkaline conditions.

In general, AEMs consist of a hydrocarbon-based polymer backbone functionalized with cationic groups. The most commonly used cationic groups are quaternary ammonium groups, because facile routes to their synthesis have already been established over the past several decades. In alkaline media, the nucleophilic attack of the hydroxide ions is well known to cause degradation of ammoniumfunctionalized AEMs. Several degradation modes have been proposed, including Hofmann elimination (E2 elimination), Ei elimination, nucleophilic substitution $\left(\mathrm{S}_{\mathrm{N}} 2\right.$ reactions) and rearrangements. ${ }^{15-17}$ The degradation of AEMs is not thought to occur through a single mechanism but rather through a combination of mechanisms; in addition, the contribution of each mechanism is thought to differ depending on the polymer structure and ammonium groups.

To improve the alkaline stability of AEMs, various cations such as guanidinium, ${ }^{18}$ imidazolium, ${ }^{19}$ pyridinium, ${ }^{20}$ sulfonium, ${ }^{21}$ phosphonium $^{22}$ and metal cations ${ }^{23}$ have been investigated. The effect of main-chain structures ranging from aliphatic ${ }^{24,25}$ to aromatic ${ }^{26,27}$ polymers has also been examined. The electronic and steric structures of the polymer backbones and the cationic moieties likely synergistically affect their alkaline stability; degradation of one component may trigger the degradation of the other. ${ }^{28,29}$ These previously published results indicate that the alkaline stability of AEMs should be investigated in a systematic manner.

This review highlights our recent studies on AEMs for FC applications. ${ }^{30-34}$ Because the anion conductivity of AEMs is the most relevant property in this application, our discussion begins with the effect of the cardo-fluorene structure in a polymer's main chain, which is a well-established building block for PEMs, on the polymer's conduction properties. We then assess the effect of the sequence of the polymer's main chain (random or block) on the conductivity of AEMs. The performance of our AEMs in a noble-metal-free FC with hydrazine as a fuel is also discussed. To improve the chemical stability of polymer backbones in alkaline and aqueous hydrazine, AEMs based on partially fluorinated poly(arylene ether) block copolymers have been examined. Further, the effect of quaternized ammonium groups, such as aliphatic ammonium, imidazolium and pyridinium ions, on the properties of AEMs is discussed in the last part of this review.

${ }^{1}$ Fuel Cells Nanomaterials Center, University of Yamanashi, Yamanashi, Japan; ${ }^{2}$ JST CREST, Kawaguchi, Saitama, Japan and ${ }^{3}$ Clean Energy Research Center, University of Yamanashi, Yamanashi, Japan

Correspondence: Professor K Miyatake, Fuel Cells Nanomaterials Center, University of Yamanashi, 4 Takeda, Kofu, Yamanashi 400-8510, Japan.

E-mail: miyatake@yamanashi.ac.jp

Received 24 March 2014; revised 21 April 2014; accepted 9 May 2014; published online 16 July 2014 


\section{EFFECT OF CARDO-FLUORENE STRUCTURE ON AEM PROPERTIES}

Although a number of polymer backbones have been studied for use in AEMs, cardo-structures such as biphenyl fluorene groups have been scarcely examined. In the past decade, we have developed various sulfonated aromatic polymers with biphenyl fluorene groups and have observed that they are suitable building blocks for PEM materials from the point of view of chemical, thermal and mechanical stability as well as proton conductivity. ${ }^{35-38}$ The three-dimensionally and densely located sulfonic acid groups on biphenyl fluorene moieties might be responsible for their high proton conductivity. These PEM research efforts prompted us to investigate the effect of cardo-fluorene groups on AEMs, because the conduction mechanism of hydroxide ions, which is based on molecular diffusion (vehicle mechanism) and/or structure reorientation (Grotthuss mechanism), is likely similar to that of protons, whereas the opposite is true in the case of the electric charge of the carrier ions.

For this purpose, we first synthesized two types of cardo-fluorenecontaining quaternized multiblock copolymers (QPEs), the main chains of which consist of diphenyl sulfone and benzophenone (QPE-1) or perfluorobiphenylene (QPE-2) (Scheme 1). Both QPEs were prepared in a similar manner: nucleophilic substitution polycondensation and Friedel-Crafts chloromethylation followed by quaternization reactions. Notably, the molecular weight as well as the chemical structure of the resulting membranes affected their mechanical strength. In the case of poly(arylene ether)-based AEMs, $M_{\mathrm{w}}$ values $>100 \mathrm{kDa}$ (determined by gel permeation chromatography on instruments calibrated with polystyrene standards) were often needed for precursor polymers to obtain self-standing membranes by solution casting. Although the Friedel-Crafts chloromethylation reaction using chloromethyl methyl ether (CMME) is often accompanied by undesirable cross-linking, the degree and position of the chloromethylation reaction could be controlled through careful optimization of the reaction conditions (for example, the amount of Lewis acid catalyst and reagents, the reaction temperature and the reaction time). For both precursor polymers (PE-1 and PE-2), the optimum conditions were $35^{\circ} \mathrm{C}$, an equimolar amount of zinc chloride and 40 equivalents of CMME relative to the molar amount of the polymer repeating unit. Under the optimized conditions, the degree of chloromethylation was controllable by simply changing the reaction time. For example, the degree of chloromethylation per fluorene unit $(x)$ was as high as 1.80 for PE-1 (reaction time $=168 \mathrm{~h}$ ), which corresponded to a high ion-exchange capacity (IEC) of $2.54 \mathrm{meq} \mathrm{g}^{-1}$ if the subsequent quaternization reaction was assumed to be quantitative. However, $x$ was as high as 1.17 (IEC $=1.56$ meq $^{-1}$ ) for PE-2 (reaction time $=96 \mathrm{~h}$ ); further reaction (at higher temperatures and with a longer reaction time) failed and gave insoluble gel products.

Chloromethylated copolymer (CMPE)-1 and CMPE-2 provided colorless, transparent, tough and ductile membranes (ca. $50 \mu \mathrm{m}$ thickness) when cast from 1,1,2,2-tetrachloroethane solutions. The obtained CMPE- 1 and CMPE- 2 membranes were then quaternized by treatment with trimethylamine aqueous solution. Finally, the immersion of the obtained QPE $\left(\mathrm{Cl}^{-}\right.$form $)$membranes in $1 \mathrm{M}$ sodium hydroxide aqueous solution provided $\mathrm{QPE}\left(\mathrm{OH}^{-}\right.$form $)$membranes. The QPE-1 and QPE-2 membranes were slightly yellow and were less soluble than the parent CMPEs. ${ }^{1} \mathrm{H}$ NMR spectra of QPE-1 (IEC as high as $1.88 \mathrm{meq} \mathrm{g}^{-1}$ ) and QPE-2 (IEC as high as $1.56 \mathrm{meq} \mathrm{g}^{-1}$ ) in DMSO- $d_{6}$ revealed that the quaternization reaction proceeded quantitatively.

The water uptake and hydroxide ion conductivity of QPE-1 membranes were mainly dependent on their IEC values. As shown in Figure 1, the higher IEC membranes exhibited higher water uptake and conductivity. The QPE-1 membrane with the highest IEC value
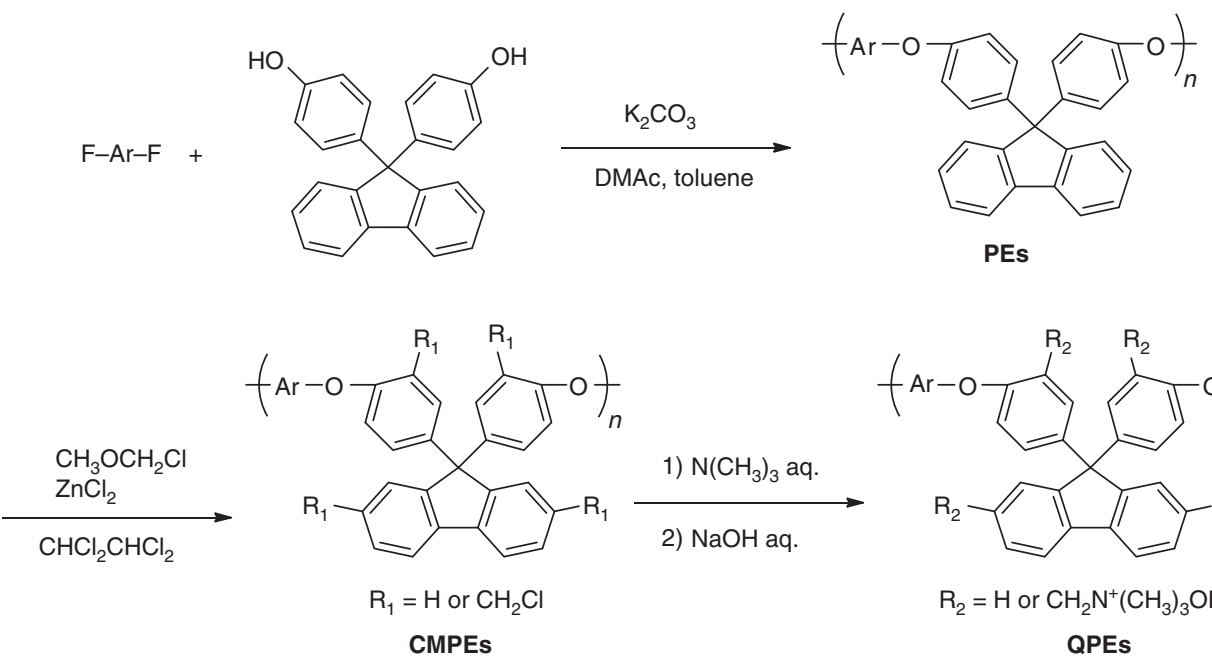

CMPEs
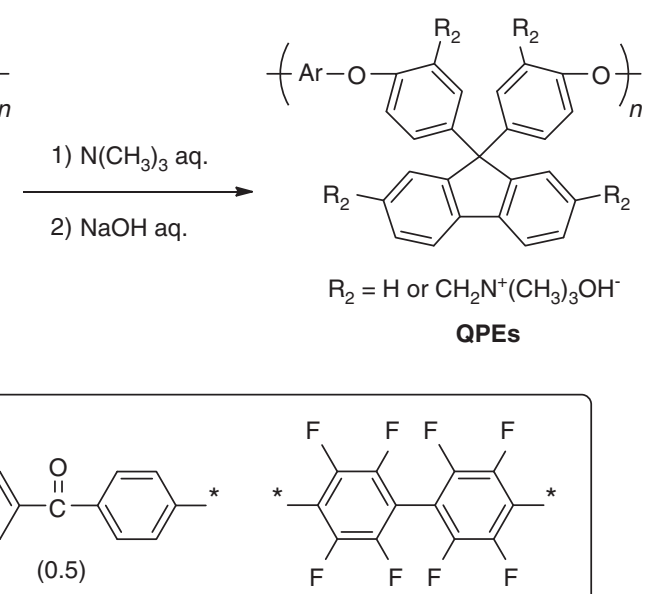

2

Scheme 1 Synthesis of cardo-fluorene-containing random QPE-1 and QPE-2. 


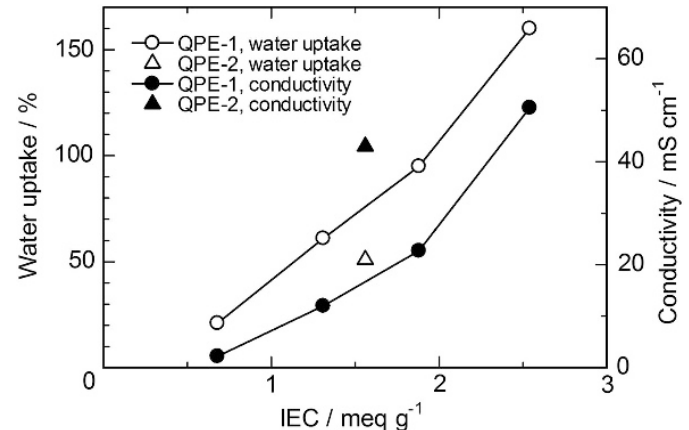

Figure 1 Water uptake and hydroxide ion conductivity as a function of IEC at $30^{\circ} \mathrm{C}$.

$\left(2.54 \mathrm{meqg}^{-1}\right)$ exhibited very high hydroxide ion conductivity $\left(50 \mathrm{mS} \mathrm{cm}^{-1}\right.$ at $30^{\circ} \mathrm{C}, 78 \mathrm{mS} \mathrm{cm}^{-1}$ at $\left.60^{\circ} \mathrm{C}\right)$. The partially fluorinated QPE-2 membrane $\left(\right.$ IEC $=1.56 \mathrm{meq}^{-1}$ ) exhibited lower water uptake $(51 \%)$ but higher hydroxide ion conductivity $\left(43 \mathrm{mS} \mathrm{cm}^{-1}\right)$ than the non-fluorinated QPE-1 membrane. We assumed that the partially fluorinated main-chain structure of the QPE-2 membrane would enhance the hydrophilic/hydrophobic differences between the polymer main chains and the ammonium-containing fluorenyl groups, resulting in better utilization of water for hydroxide ion conduction. The effect of partial fluorination on the properties of the membrane is discussed in more detail for the block copolymer membranes in the subsequent section.

\section{EFFECT OF THE MAIN-CHAIN SEQUENCE (RANDOM OR BLOCK) ON AEM PROPERTIES}

In the pursuit of our aromatic PEM studies, we demonstrated that multiblock copolymers with highly localized and dense sulfonic acid groups in the hydrophilic blocks exhibited much higher proton conductivity compared with the random copolymers because of their unique phase-separated morphology with well-interconnected proton transport pathways. ${ }^{38-42}$ Biphenyl fluorene groups serve well as scaffolds for sulfonic acid groups. These results prompted us to replace sulfonic acid groups with ammonium groups in the multiblock copolymers to achieve better-performing AEMs (Scheme 2).

A series of precursor multiblock poly(arylene ether)s, PE-bl-1 (X8Y8), (X16Y11), (X22Y11) and (X22Y23) (the numbers after X and $\mathrm{Y}$ represent the degrees of polymerization of the hydrophobic and fluorene-containing (later hydrophilic) blocks, respectively), were synthesized by nucleophilic substitution polycondensation of separately synthesized oligomers. The following chloromethylation reaction conditions were similar to but slightly modified from those for the aforementioned polymers (PE-1 and PE-2). For PE-bl-1, the optimum conditions were $45^{\circ} \mathrm{C}$, an equimolar amount of zinc chloride, and 80 equivalents of CMME relative to the molar amount of the polymer repeating unit. Because the block copolymers exhibited better solubility, more severe reaction conditions were used for the block copolymers compared with those used for PE-1 and PE-2. Accordingly, a higher degree of chloromethylation was achieved for the multiblock copolymers. For QPE-bl-1 (X16Y11), the highest degree of chloromethylation was 2.78 per repeat unit by $144 \mathrm{~h}$ of reaction (Figure 2a). The fluorene groups were more reactive than the main chains because of the lack of electron-withdrawing ketone and sulfone groups (Figure 2b). The fluorene groups were selectively and quantitatively chloromethylated at the 2 and 7 positions, whereas the number of chloromethyl groups in the main chains did not exceed one, even after $144 \mathrm{~h}$ of reaction. The total degree of chloromethylation of multiblock PE-bl-1 was much higher than that of random PE-1 under the same reaction conditions. Notably, QPE-bl-1 with a high degree of chloromethylation (2.81 per fluorene moiety, X22Y11) retained good solubility, which enabled tough membranes to be prepared by casting from the solution. The quaternization and the subsequent anion-exchange reactions of CMPE-bl-1 membranes afforded the target QPE-bl-1 membranes in $\mathrm{OH}^{-}$form. The QPE-bl-1 membranes were transparent and pale yellow, and they exhibited lower solubility in organic solvents than the membranes of the parent CMPE-bl-1. The QPE-bl-1 membranes in the $\mathrm{OH}^{-}$form were soluble in DMSO and those in the $\mathrm{Cl}^{-}$form were soluble in DMF and DMAc when their IEC was $<1.5$ meq $^{-1}$. The multiblock QPE-bl-1 exhibited better solubility than the random QPE-1; the random QPE-1 in the $\mathrm{OH}^{-}$form was also soluble in DMSO but was not soluble in the $\mathrm{Cl}^{-}$form in any of the examined solvents.

To analyze the effect of sequence structure on morphology, scanning transmission electron microscopic (STEM) images were taken of the multiblock QPE-bl-1 membrane and were subsequently compared with those of the random QPE-1 membrane. Figures 3a and $\mathrm{b}$ show STEM images of the QPE-bl-1 (X16Y11) $(\mathrm{IEC}=1.52$ meq $\left.\mathrm{g}^{-1}\right)$ and QPE-1 $\left(\right.$ IEC $\left.=1.88 \mathrm{meq}^{-1}\right)$ membranes stained with tungstate ions. Whereas the random copolymer QPE-1 membrane showed no distinct phase separation, the block copolymer QPE-bl-1 (X16Y11) membrane showed a phase-separated morphology with dark domains (ammonium-containing hydrophilic moieties approximately $5 \mathrm{~nm}$ wide) throughout the field of view. We assumed that the developed hydrophilic/hydrophobic phase separation was caused by the sequential block structure, despite the lower IEC value or lower ionic concentration of multiblock QPE-bl-1 compared with the random QPE-1.

Water uptake $\left(30^{\circ} \mathrm{C}\right)$ and hydroxide ion conductivities $\left(40^{\circ} \mathrm{C}\right)$ of the QPE-bl-1 membranes were measured in water and are plotted as a function of IEC in Figure 4. Water uptake of the QPE-bl-1 membranes increased with increasing IEC. A comparison of multiblock QPE-bl-1 and random QPE-1 membranes revealed that the multiblock copolymer membranes absorbed less water, except in the case of the QPE-bl-1 (X8Y8) membranes. These results indicate that multiblock copolymer AEMs with longer hydrophilic or hydrophobic segments tend to have more developed hydrophilic/hydrophobic phase-separated morphology, resulting in the suppression of excessive water uptake and swelling.

Similar to the water uptake, the hydroxide ion conductivity of the multiblock copolymer QPE membranes increased with increasing IEC. The conductivities of the three QPE-bl-1 membranes, X16Y11, $\mathrm{X} 22 \mathrm{Y} 11$ and $\mathrm{X} 22 \mathrm{Y} 23$, had a similar dependence on their IEC values. The QPE-bl-1 (X16Y11) membrane with IEC $=1.93$ meqg $^{-1}$ exhibited the highest conductivity $\left(96 \mathrm{mS} \mathrm{cm}^{-1}\right)$. The temperature dependence of the hydroxide ion conductivities of the QPE-bl-1 (X16Y11) and QPE-1 membranes in water is compared in Figure 5. Similar to the results in Figure 4, the conductivities followed the order of the IEC values for the same series of QPE-bl-1 and QPE-1. The QPE-bl-1 (X16Y11) membrane $\left(\right.$ IEC $=1.93 \mathrm{meqg}^{-1}$ ) exhibited the highest conductivity $\left(144 \mathrm{mS} \mathrm{cm}^{-1}\right)$ at $80^{\circ} \mathrm{C}$, which was ca. 3.2 times higher than that of the QPE-1 membrane with a similar IEC $\left(1.88 \mathrm{meq}^{-1}\right)$ under the same conditions. The important point is that the multiblock copolymer QPE membranes exhibited decreased water uptake but considerably higher conductivity compared with the random copolymer QPE membranes. These results suggest that our method of preparing multiblock copolymer structures with highly ionized hydrophilic blocks effectively resulted in improved ionic conductivity 
a
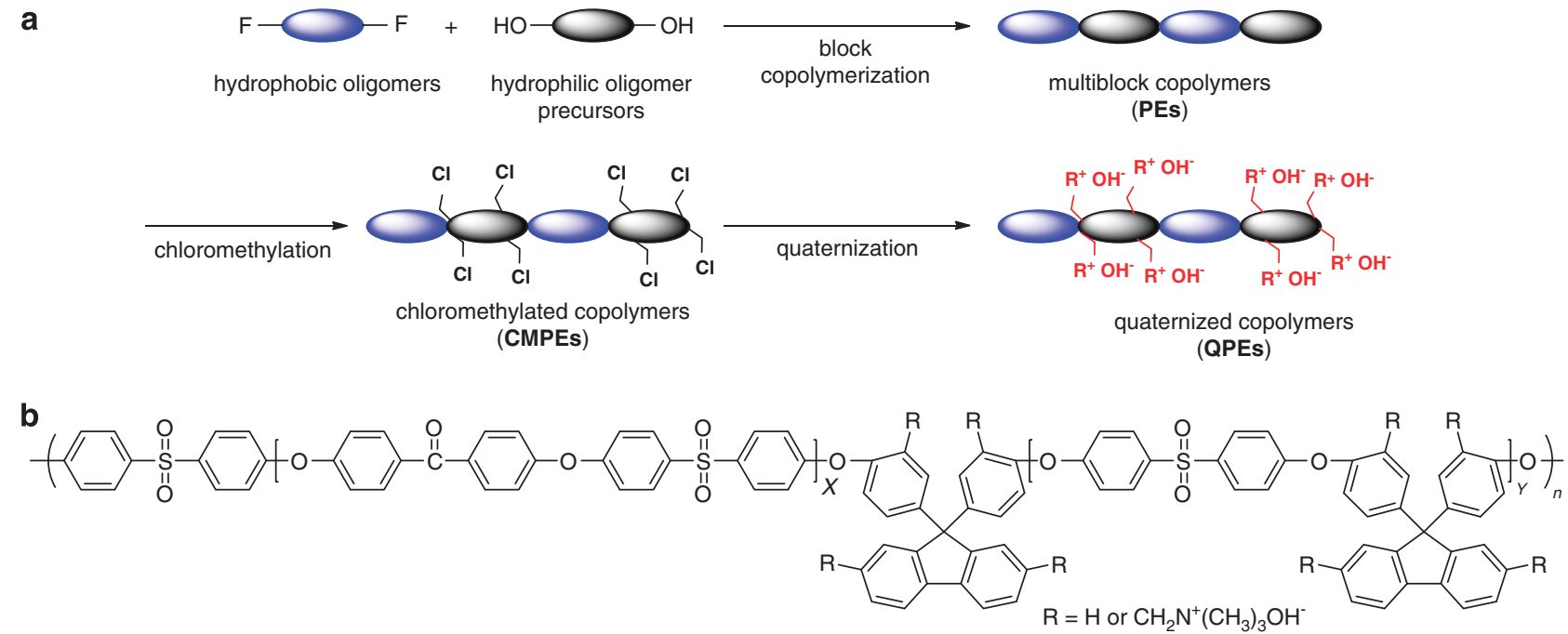

QPE-bl-1

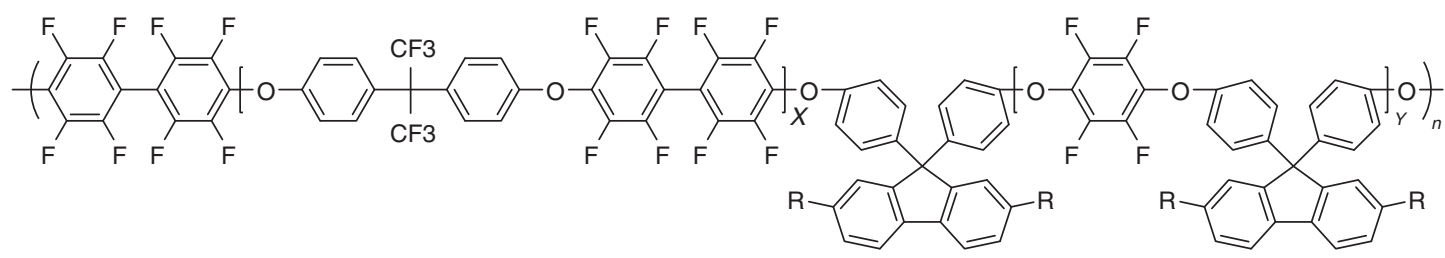

QPE-bl-2

$\mathrm{R}=\mathrm{H}$ or $\mathrm{CH}_{2} \mathrm{~N}^{+}\left(\mathrm{CH}_{3}\right)_{3} \mathrm{OH}^{-}$

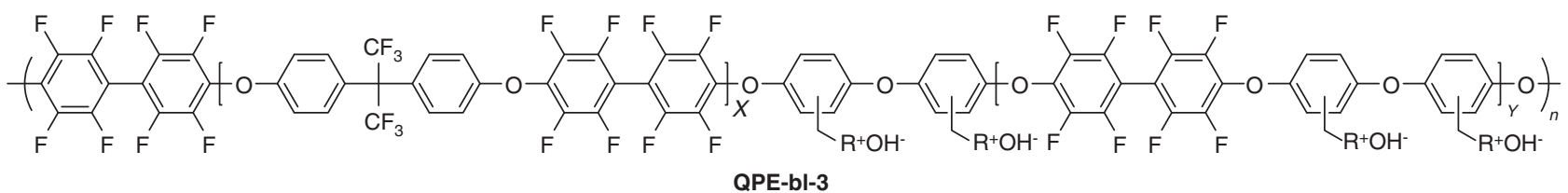

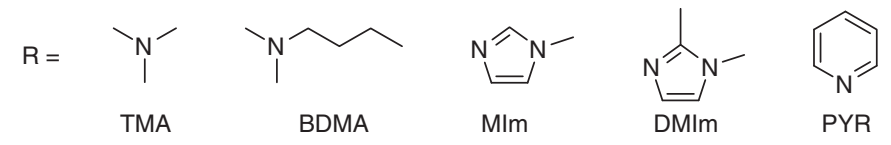

Scheme 2 (a) Conceptual scheme of the quaternized multiblock copolymers and (b) structure of the quaternized multiblock copolymers QPE-bl-1, QPE-bl-2 and QPE-bl-3.

of the resulting AEMs, as already demonstrated for PEMs. We have demonstrated that the well-developed nanophase-separated morphology with interconnected ionic channels contributed to the effective use of water for ion conduction. Furthermore, we have successfully operated a noble-metal-free FC fabricated using a QPE-bl-1 (X16Y11) $\left(\right.$ IEC $\left.=1.93 \mathrm{meq} \mathrm{g}^{-1}\right)$ membrane, in which $\mathrm{Ni}$ and Co were used as the anode and cathode, respectively, and hydrazine (in $\mathrm{KOH}$ aqueous solution) was used as a fuel. Reasonably good FC performance was obtained, with a maximum power density of $161 \mathrm{~mW} \mathrm{~cm}^{-2}$ at a current density of $446 \mathrm{~mA} \mathrm{~cm}^{-2}$ with air and a maximum power density of $297 \mathrm{~mW} \mathrm{~cm}^{-2}$ at a current density of $826 \mathrm{mAcm}^{-2}$ with oxygen.

\section{PARTIALLY FLUORINATED QPE-BL-2}

Poly(arylene ether sulfone), which is one of the most commonly used polymer backbones for both PEMs and AEMs, suffers from mainchain degradation in alkaline media. ${ }^{29}$ Because alkaline stability is one of the most important features of AEMs, we have developed an advanced version of multiblock copolymer QPEs by replacing sulfone (and ketone) groups with perfluoroaromatic groups to improve both their conductivity properties and their chemical stability (QPE-bl-2). Similar to the aforementioned QPE-bl-1, a series of QPE-bl-2 were obtained by block copolymerization, chloromethylation and quaternization, followed by an anion-exchange reaction. Under the optimized chloromethylation reaction conditions for PE-bl-2, the highest degree of chloromethylation was approximately two per repeat unit by $48 \mathrm{~h}$ of reaction. Interestingly, in the case of partially fluorinated PE-bl-2, the chloromethyl groups were not substituted at the main chain; rather, they were substituted only at the fluorenyl groups. These results differed from those of non-fluorinated polymers (PE-bl-1), where the chloromethyl groups were substituted at both the polymer main chain and the fluorenyl groups. Steric hindrance as well as the electron-withdrawing nature of the fluorine groups attached ortho to the ether bonds is presumably responsible for the inactivity at the polymer main chain. The solution casting of CMPEbl-2 followed by quaternization with trimethylamine was conducted in a manner similar to that used for the aforementioned polymers to obtain the targeted partially fluorinated QPE-bl-2 membranes. The obtained QPE-bl-2 membranes were light brown and were insoluble in all of the evaluated solvents. 

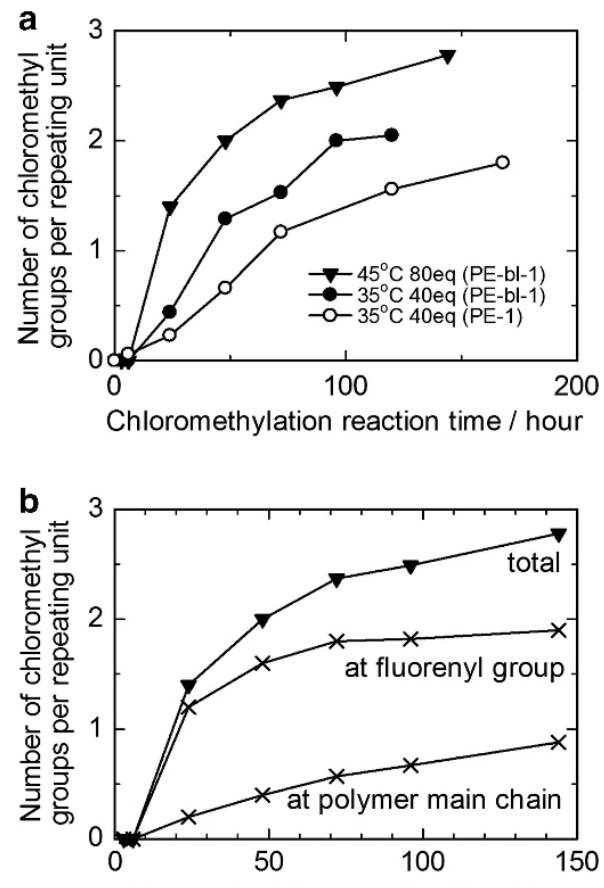

Chloromethylation reaction time / hour

Figure 2 Time dependence of the degree of chloromethylation (a) for block PE-bl-1 (X16Y11) and random PE-1 and (b) for block PE-bl-1 (X16Y11) at the fluorenyl group and main chain at $45^{\circ} \mathrm{C}$ and 80 equivalents of $\mathrm{CMME}$.

Figure 3c shows a TEM image of QPE-bl-2 (X16Y12) $($ IEC $=1.3$ meq $\mathrm{g}^{-1}$ ) stained with tetrachloroplatinate ions. The hydrophilic domains were approximately $4-8 \mathrm{~nm}$ wide and were interconnected. The interface between the hydrophilic and hydrophobic parts was somewhat more distinct than that of QPE-bl-1. However, a detailed discussion of the effect of partial fluorination on morphology is not valid because the counter anions differ (that is, tungstate in the case of QPE-bl-1 and tetrachloroplatinate in the case of QPE-bl-2). Nevertheless, the sequenced structures obviously promote the hydrophilic/hydrophobic phase separation.

The water uptake of the QPE-2 membranes is included in Figure 4a. Because the water uptake of QPE-bl-2 was similar to that of QPE-bl-1, the partial fluorination in the main chains is unlikely to affect the hydrophilicity of the bulk membrane. These results might be reasonable, because water is absorbed mainly by the hydrophilic ammonium groups distanced from the main chains. Similar to their water uptake behavior, the QPE-bl-2 and QPE-bl-1 membranes exhibited comparable hydroxide ion conductivities, as shown in Figure $4 \mathrm{~b}$.

The alkaline stability of the QPE-bl-2 membranes was tested under severe conditions in an aqueous $\mathrm{KOH}$ solution containing hydrazine at $80^{\circ} \mathrm{C}$. After a $500-\mathrm{h}$ soaking test, the QPE-bl-2 membrane $\left(\right.$ IEC $\left.=1.3 \mathrm{meq}^{-1}\right)$ exhibited very minor weight losses while maintaining its toughness and flexibility. In comparison, the QPE-bl-1 membrane became brittle under the same testing conditions. The partial fluorination substantially improved the alkaline stability of the polymer main chain. In contrast, the water uptake decreased to $42 \%$ after $500 \mathrm{~h}$ of testing, indicating the decomposition of the hydrophilic ammonium groups. To further investigate the decomposition of the ammonium groups, the hydroxide ion conductivity was compared with that of the pristine membrane (Figure 6). The hydroxide ion conductivity decreased with increasing testing time; the conductivity
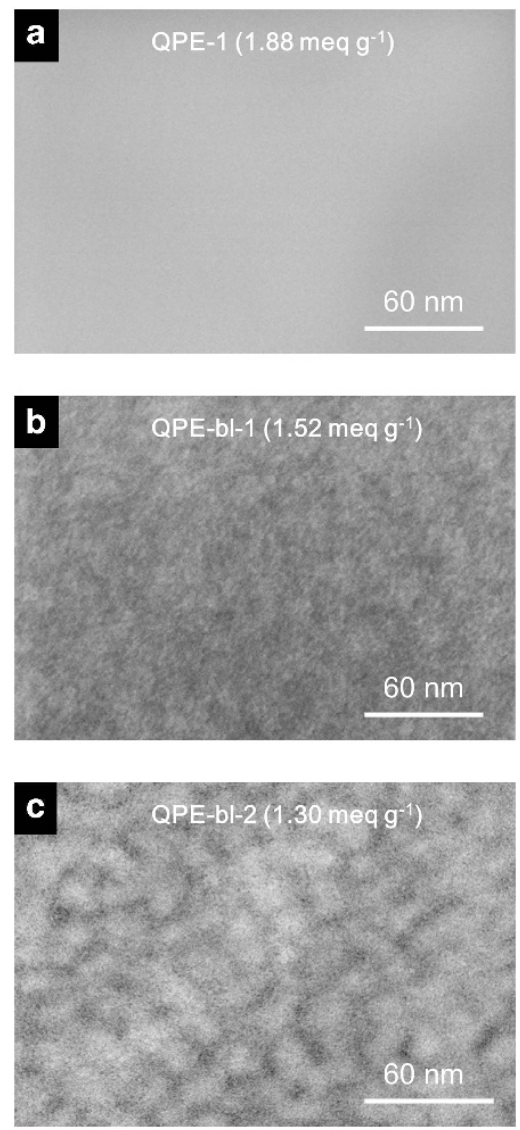

Figure 3 STEM images of (a) QPE-1 (IEC $=1.88$ meq $^{-1}$ ) and (b) QPE-bl-1 (X16Y11) $\quad\left(I E C=1.52 \mathrm{meqg}^{-1}\right) \quad$ stained with tungstate ions and (c) QPE-bl-2 (X16Y12) membrane $\left(\mathrm{IEC}=1.30 \mathrm{meqg}^{-1}\right)$ stained with tetrachloroplatinate ions.

at $60{ }^{\circ} \mathrm{C}$ decreased from 37 (initial) to $0.41 \mathrm{mS} \mathrm{cm}^{-1}$ (after $500 \mathrm{~h}$ ). The apparent activation energies for the hydroxide ion conduction were similar: 12.0 (pristine), $14.1(24 \mathrm{~h}), 15.3(200 \mathrm{~h})$, and $11.7 \mathrm{~kJ} \mathrm{~mol}^{-1}(500 \mathrm{~h})$. These results indicate that the loss of ammonium groups did not alter the anion conduction mechanism in the membranes.

\section{EFFECT OF AMMONIUM GROUPS}

As previously described, we observed that the introduction of fluorine atoms into the polymer main chain improved the alkaline stability of the membranes; however, the trimethylbenzyl ammonium groups were unstable. To improve the alkaline stability of ammonium groups in AEMs, we investigated the stability of five different ammonium groups: trimethyl ammonium (TMA) and $\mathrm{N}$-butyldimethyl ammonium (BDMA) (aliphatic amine types) and 1-methylimidazolium (MIm), 1,2-dimethylimidazolium (DMIm), and pyridinium (PYR) (aromatic amine types). These ammonium groups were designed to be connected at the benzyl position of the aromatic polymer main chain. For the hydrophilic parts of the polymer main chain, we used diphenyl ether instead of biphenyl fluorene groups as a scaffold for the ionic functionalization.

We prepared the QPE-bl-3 membranes by treating the precursor CMPE-bl-3 membranes with amines. The quaternization reaction conditions depend on the basicity, nucleophilicity and steric nature of the amines. Therefore, we first investigated the reactivity of various amines with a chloromethylated model compound and observed that 

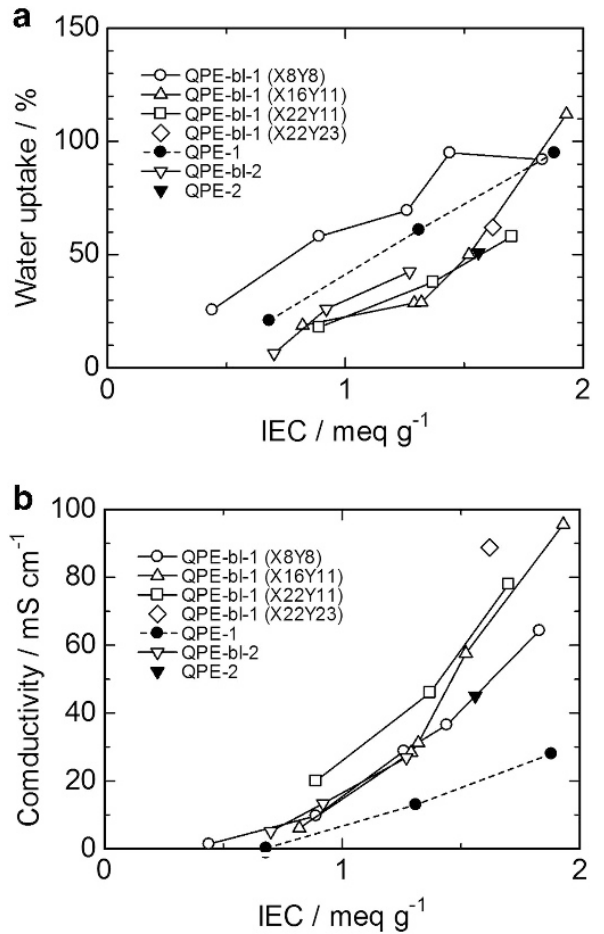

Figure 4 (a) Water uptake at $30^{\circ} \mathrm{C}$ and (b) hydroxide ion conductivity at $40^{\circ} \mathrm{C}$ of QPE membranes as a function of their IEC.

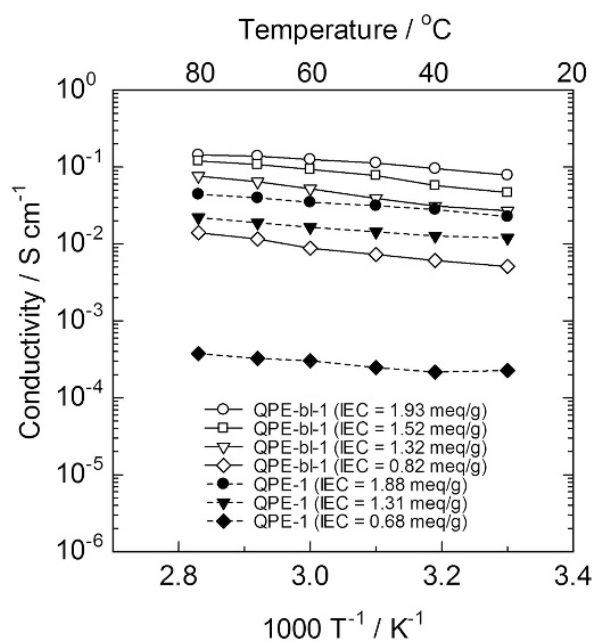

Figure 5 Temperature dependence of the hydroxide ion conductivities of multiblock QPE-bl-1 (X16Y11) and random QPE-1 membranes.

a higher reaction temperature was needed for sterically hindered or conjugated amines, such as BDMA $\left(60^{\circ} \mathrm{C}\right)$, DMIm $\left(80^{\circ} \mathrm{C}\right)$ and PYR $\left(80^{\circ} \mathrm{C}\right)$, to complete the quaternization reaction within $48 \mathrm{~h}$. The reaction conditions for the model compound were applied to the polymer reaction; however, PYR required a higher reaction temperature $\left(100{ }^{\circ} \mathrm{C}\right)$, most likely because its polymer structure is more sterically hindered than that of the model compound.

In all cases, quantitative conversion of the chloromethyl groups to the corresponding ammonium groups was achieved. Unlike the aforementioned QPE-bl-1 and QPE-bl-2, which exhibited poor solubility after the quaternization, the obtained QPE-bl-3 ( $\mathrm{Cl}^{-}$form) was soluble in polar aprotic solvents and could form transparent and

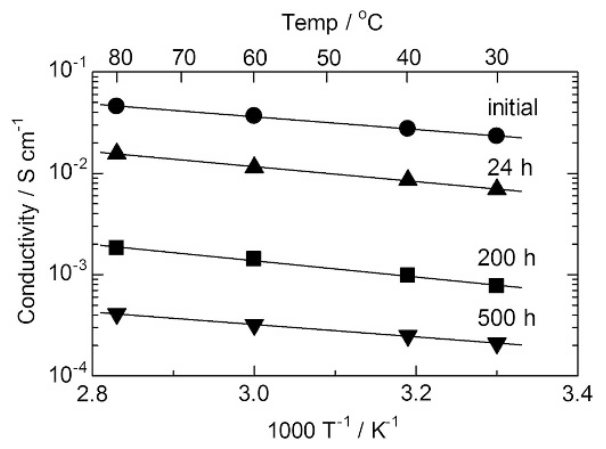

Figure 6 Changes in the hydroxide ion conductivity of the QPE-bl-2 (X16Y12) membrane $\left(\mathrm{IEC}=1.3 \mathrm{meqg}^{-1}\right)$ during the stability test in $1 \mathrm{M}$ aqueous $\mathrm{KOH}$ solution containing 5 wt $\%$ hydrazine at $80^{\circ} \mathrm{C}$.

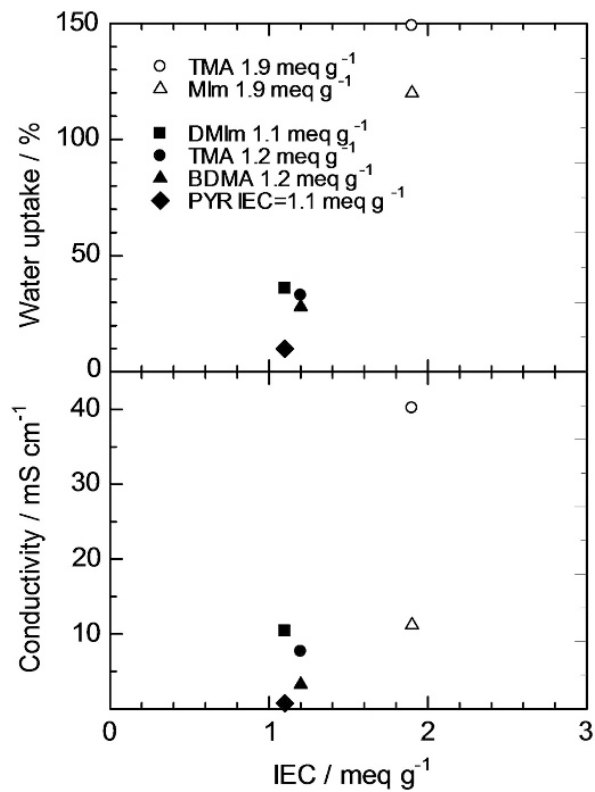

Figure 7 Water uptake $\left(30^{\circ} \mathrm{C}\right)$ and hydroxide ion conductivity $\left(40^{\circ} \mathrm{C}\right)$ of QPE-bl-3 membranes as a function of their IEC.

ductile membranes when cast from DMAc solution. As expected, the higher IEC membranes exhibited greater water uptake. In QPE-bl-3 (X5Y9), the water uptake of TMA (149\%) was greater than that of MIm (120\%), most likely because the benzyltrimethylammonium groups, as smaller cations, have better access to water molecules compared with the benzylimidazolium groups.

Figure 7 shows the water uptake at $30^{\circ} \mathrm{C}$ and hydroxide ion conductivity in water at $40^{\circ} \mathrm{C}$ as a function of IEC values for QPE-bl-3 membranes with different ammonium groups. The general trend was similar to that observed for the aforementioned membranes: the membranes with higher IEC values exhibited greater water uptake and greater hydroxide ion conductivity. A comparison of the five types of ammonium groups indicated that the hydrophobicity of the ion-exchange groups tended to decrease the hydroxide ion conductivity as well as the water uptake. For example, TMAand BDMA-QPE membranes exhibit comparable IEC values $\left(1.2\right.$ meq $\left.^{-1}\right)$; however, the more hydrophobic BDMA-QPE membrane exhibited lower water uptake and lower ion conductivity.

Figure 8 shows the temperature dependence of the hydroxide ion conductivity in water for QPE-bl-3 membranes with different 
ammonium groups. The conductivities of the QPE-bl-3 membranes displayed an approximate Arrhenius-type temperature dependence from 30 to $80^{\circ} \mathrm{C}$, and the apparent activation energies were $12-15 \mathrm{~kJ} \mathrm{~mol}^{-1}$. These values were comparable to those for the aforementioned QPE membranes, suggesting that they share a similar ion conduction mechanism that is independent of the type of ionexchange groups, the IEC values and the polymer main-chain sequence (random or block).

The alkaline stability of the QPE-bl-3 membranes was investigated in an aqueous solution of $1 \mathrm{M}$ potassium hydroxide at $80^{\circ} \mathrm{C}$ for 24 and $100 \mathrm{~h}$ (Figure 9a). Among the five QPE membranes, PYR exhibited the greatest alkaline stability, with minor decreases in its conductivity, water uptake and dry weight within $24 \mathrm{~h}$. Although the difference in the water uptake values was not negligible, we considered

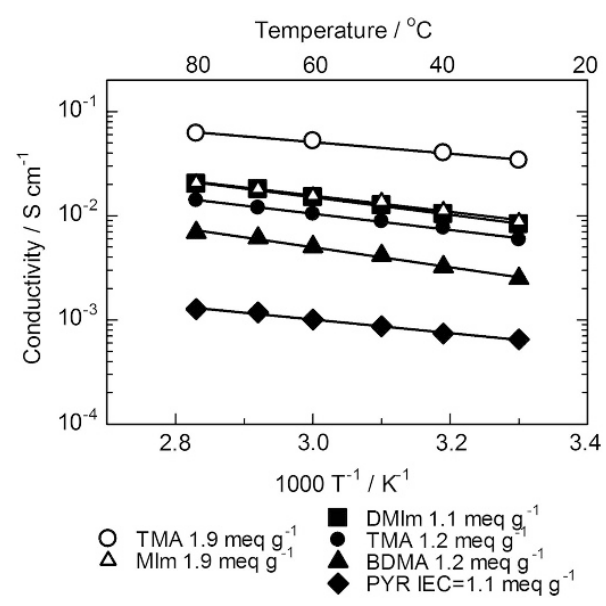

Figure 8 Temperature dependence of the hydroxide ion conductivities of multiblock copolymer QPE-bl-3 membranes. that the ion-exchange groups with high basicity and delocalized cations (in conjugated rings) are less likely to undergo nucleophilic attack by the hydroxide ions. DMIm exhibited greater alkaline stability than MIm, which also supports this hypothesis. DMIm is advantageous in terms of the steric hindrance and hydrophobicity of methyl substituents on the planar structure of the imidazolium ring. The ion conductivity of the five QPE membranes decreased under the tested conditions, indicating that both the aliphatic and aromatic ammonium groups degrade in strong alkaline solutions at high temperatures.

We subsequently examined the stability of the QPE-bl-3 membranes in $10 \%$ hydrazine aqueous solution at $80^{\circ} \mathrm{C}$ (Figure 9b). Similar to what was observed with the $\mathrm{KOH}$ solution, PYR was the most stable of the QPE-bl-3 membranes under the tested conditions and retained a high conductivity for $24 \mathrm{~h}$. We found that hydrazine causes more serious degradation of the QPE membranes than aqueous $\mathrm{KOH}$ in terms of their weight, conductivity, water uptake and mechanical strength. The posttest samples were insoluble in all the investigated solvents, which precluded NMR analyses. Whereas the IR spectra suggested that only minor structural changes occurred in the polymer main chains, the lack of ductility and mechanical strength in the posttest samples is indicative of degradation of the main chain and decomposition of the ammonium groups.

\section{SUMMARY AND OUTLOOK}

We have developed a novel series of AEMs for alkaline FC applications. For alkaline FCs that use hydrazine as a fuel, we have examined the effect of the chemical structures of the ammonium-functionalized aromatic polymers on stability and anion conductivity. First, we observed that cardo-structures such as biphenyl fluorene groups serve well as scaffolds for the ammonium groups in AEMs. In addition, the polymer main-chain sequence affected the anion conductivity: multiblock copolymer AEMs exhibited greater anion conductivity
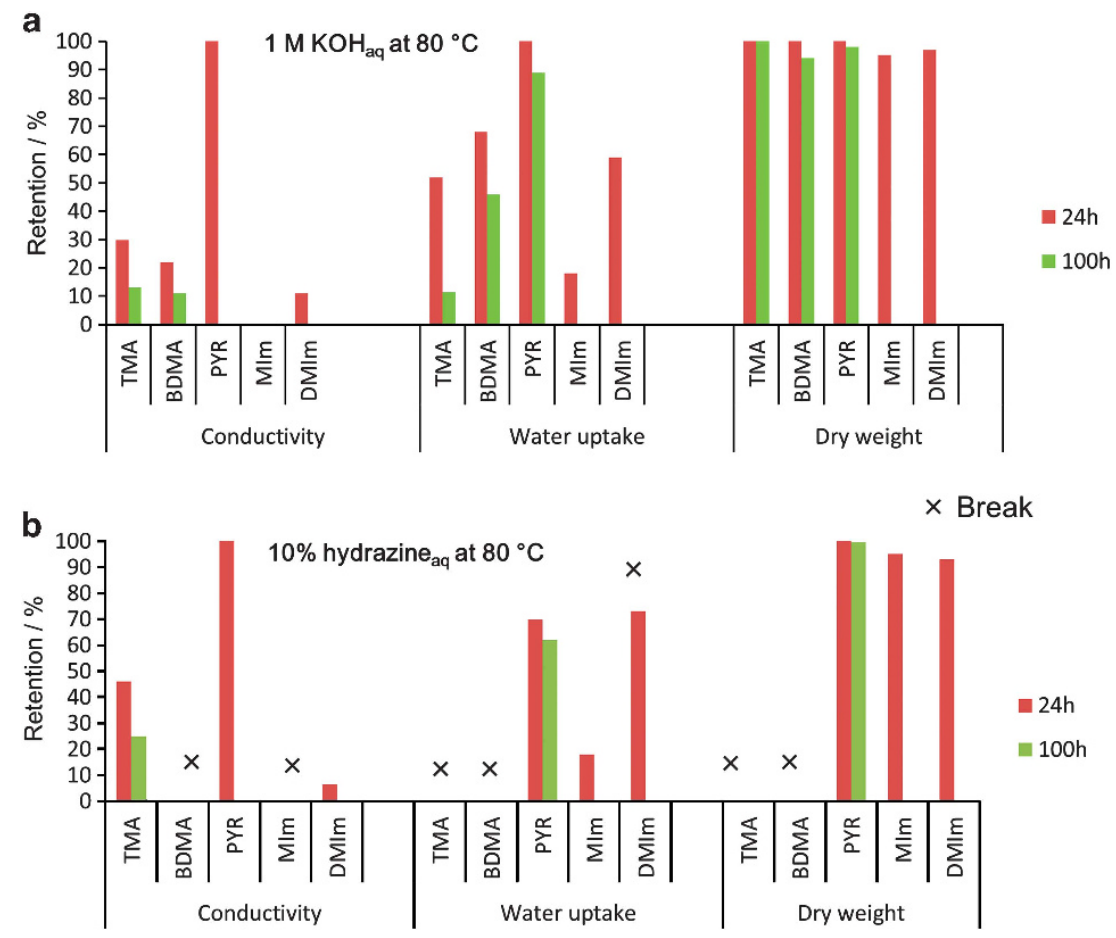

Figure 9 Changes in hydroxide ion conductivity, water uptake and weight of QPE-bl-3 membranes in the accelerated (a) $\mathrm{KOH}$ and (b) hydrazine stability tests. 
than their random copolymer analogues, which is a phenomenon similar to that observed for proton-conductive membranes. Furthermore, partial fluorination in the main chain was observed to be effective in improving the alkaline stability of the membranes. We subsequently evaluated the alkaline stability of various ammonium groups and observed that pyridinium groups are apparently more stable than typical aliphatic ammonium groups (for example, trimethylammonium).

The results described herein indicate that the aromatic AEMs are potentially applicable to alkaline FCs that use hydrogen or other fuels, such as hydrazine. However, there are still issues to be resolved for practical alkaline FC applications. In particular, the alkaline stability of ammonium groups as well as that of the polymer main chain is considered to be the most important issue. We believe that further molecular design of ammonium groups as well as further design of polymer main chains will facilitate the development of highperformance and robust AEMs or AEMFCs.

\section{ACKNOWLEDGEMENTS}

This work was partly supported by the Ministry of Education, Culture, Sports, Science and Technology of Japan through a Grant-in-Aid for Scientific Research (23350089).

1 Varcoe, J. R. \& Slade, R. C. T. Prospects for alkaline anion-exchange membranes in low temperature fuel cells. Fuel Cells 5, 187-200 (2005).

2 Pan, J., Chen, C., Zhuang, L. \& Lu, J. Designing advanced alkaline polymer electrolytes for fuel cell applications. Acc. Chem. Res. 45, 473-481 (2012).

3 Leng, Y., Chen, G., Mendoza, A. J., Tighe, T. B., Hickner, M. A. \& Wang, C.-Y. Solid-state water electrolysis with an alkaline membrane. J. Am. Chem. Soc. 134, 9054-9057 (2012).

4 Pavel, C. C., Cecconi, F., Emiliani, C., Santiccioli, S., Scaffidi, A., Catanorchi, S. \& Comotti, M. Highly efficient platinum group metal free based membrane-electrode assembly for anion exchange membrane water electrolysis. Angew. Chem. Int. Ed. 53 (5), 1378-1381 (2014).

5 Li, X., Zhang, H., Mai, Z., Zhang, H. \& Vankelecom, I. Ion exchange membranes for vanadium redox flow battery (VRB) applications. Energy Environ. Sci. 4, 1147-1160 (2011).

6 Chen, D., Hickner, M. A., Agar, E. \& Kumbur, E. C. Optimized anion exchange membranes for vanadium redox flow batteries. ACS Appl. Mater. Interfaces 5, 7559-7566 (2013).

7 Sata, T. Studies on anion exchange membranes having permselectivity for specific anions in electrodialysis-effect of hydrophilicity of anion exchange membranes on permselectivity of anions. J. Membr. Sci. 167, 1-31 (2000).

8 Swager, T. M. The molecular wire approach to sensory signal amplification. Acc. Chem. Res. 31, 201-207 (1998).

9 Ho, H.-A., Najari, A. \& Leclerc, M. Optical detection of DNA and proteins with cationic polythiophenes. Acc. Chem. Res. 41, 168-178 (2008).

10 Hickner, M. A. Ion-containing polymers: new energy \& clean water. Mater. Today 13, 34-41 (2010).

11 Hickner, M. A., Herring, A. M. \& Coughlin, E. B. Anion exchange membranes: current status and moving forward. J. Polym. Sci. Part B Polym. Phys. 51, 1727-1735 (2013).

12 Park, C. H., Lee, C. H., Guiver, M. D. \& Lee, Y. M. Sulfonated hydrocarbon membranes for medium-temperature and low-humidity proton exchange membrane fuel cells (PEMFCs). Prog. Polym. Sci. 36, 1443-1498 (2011).

13 Zhang, H. \& Shen, P. K. Recent development of polymer electrolyte membranes for fuel cells. Chem. Rev. 112, 2780-2832 (2012).

14 Lu, S., Pan, J., Huang, A., Zhuang, L. \& Lu, J. Alkaline polymer electrolyte fuel cells completely free from noble metal catalysts. Proc. Natl Acad. Sci. USA 105, 20611-20614 (2008).

15 Couture, G., Alaaeddine, A., Boschet, F. \& Ameduri, B. Polymeric materials as anionexchange membranes for alkaline fuel cells. Prog. Polym. Sci. 36, 1521-1557 (2011).

16 Merle, G., Wessling, M. \& Nijmeijer, K. Anion exchange membranes for alkaline fuel cells: a review. J. Membr. Sci. 377, 1-35 (2011).
17 Chempath, S., Boncella, J. M., Pratt, L. R., Henson, N. \& Pivovar, B. S. Density functional theory study of degradation of tetraalkylammonium hydroxides. J. Phys. Chem. C 114, 11977-11983 (2010).

18 Zhang, Q., Li, S. \& Zhang, S. A novel guanidinium grafted poly(aryl ether sulfone) for high-performance hydroxide exchange membranes. Chem. Commun. 46, 7495-7497 (2010).

19 Lin, B., Dong, H., Li, Y., Si, Z., Gu, F. \& Yan, F. Alkaline stable C2-substituted imidazolium-based anion-exchange membranes. Chem. Mater. 25, 1858-1867 (2013).

20 Huang, A., Xia, C. Xiao, C. \& Zhuang, L. Composite anion exchange membrane for alkaline direct methanol fuel cell: structural and electrochemical characterization. J. Appl. Polym. Sci. 100, 2248-2251 (2006).

21 Zhang, B., Gu, S., Wang, J., Liu, Y., Herring, A. M. \& Yan, Y. Tertiary sulfonium as a cationic functional group for hydroxide exchange membranes. RSC Adv. 2, 12683-12685 (2012)

22 Noonan, K. J. T., Hugar, K. M., Kostalik, H. A. IV, Lobkovsky, E. B., Abruña, H. D. \& Coates, G. W. Phosphonium-functionalized polyethylene: a new class of base-stable alkaline anion exchange membranes. J. Am. Chem. Soc. 134, 18161-18164 (2012).

23 Zha, Y., Disabb-Miller, M. L., Johnson, Z. D., Hickner, M. A. \& Tew, G. N. Metal-cationbased anion exchange membranes. J. Am. Chem. Soc. 134, 4493-4496 (2012).

24 Tomoi, M., Yamaguchi, K., Ando, R., Kantake, Y., Aosaki, Y. \& Kubota, H. Synthesis and thermal stability of novel anion exchange resins with spacer chains. J. Appl. Polym. Sci. 64, 1161-1167 (1997).

25 Robertson, N. J., Kostalik, H. A IV, Clark, T J, Mutolo, P. F. Abruna, H. D. \& Coates, G. W. Tunable high performance cross-linked alkaline anion exchange membranes for fuel cell applications. J. Am. Chem. Soc. 132, 3400-3404 (2010).

26 Hibbs, M. R. Alkaline stability of poly(phenylene)-based anion exchange membranes with various cations. J. Polym. Sci. Part B Polym. Phys. 51, 1736-1742 (2013).

$27 \mathrm{Li}$, N., Leng, Y., Hickner, M. A. \& Wang, C.-Y. Highly stable, anion conductive, combshaped copolymers for alkaline fuel cells. J. Am. Chem. Soc. 135, 10124-10133 (2013).

28 Nuñez, S. A. \& Hickner, M. A. Quantitative ${ }^{1} \mathrm{H}$ NMR analysis of chemical stabilities in anion-exchange membranes. ACS Macro. Lett. 2, 49-52 (2013).

29 Arges, C. G. \& Ramani, V. Two-dimensional NMR spectroscopy reveals cation-triggered backbone degradation in polysulfone-based anion exchange membranes. Proc. Natl Acad. Sci. USA 110, 2490-2495 (2013).

30 Tanaka, M., Koike, M., Miyatake, K. \& Watanabe, M. Anion conductive aromatic ionomers containing fluorenyl groups. Macromolecules 43, 2657-2659 (2010).

31 Tanaka, M., Koike, M., Miyatake, K. \& Watanabe, M. Synthesis and properties of anion conductive ionomers containing fluorenyl groups for alkaline fuel cell applications. Polym. Chem. 2, 99-106 (2011).

32 Tanaka, M., Fukasawa, K., Nishino, E., Yamaguchi, S., Yamada, K., Tanaka, H., Bae, B., Miyatake, K. \& Watanabe, M. Anion conductive block poly(arylene ether)s: synthesis, properties, and application in alkaline fuel cells. J. Am. Chem. Soc. 133, 10646-10654 (2011).

33 Ono, H., Miyake, J., Bae, B., Watanabe, M. \& Miyatake, K. Synthesis and properties of partially fluorinated poly(arylene ether) block copolymers containing ammonium groups as anion conductive membranes. Bull. Chem. Soc. Jpn. 86, 663-670 (2013).

34 Miyake, J., Fukasawa, K., Watanabe, M. \& Miyatake, K. Effect of ammonium groups on the properties and alkaline stability of poly(arylene ether)-based anion exchange membranes. J. Polym. Sci. Part A Polym. Chem. 52, 383-389 (2014).

35 Miyatake, K., Chikashige, Y., Higuchi, E. \& Watanabe, M. Tuned polymer electrolyte membranes based on aromatic polyethers for fuel cell applications. J. Am. Chem. Soc. 129, 3879-3887 (2007)

36 Bae, B., Miyatake, K. \& Watanabe, M. Effect of the hydrophobic component on the properties of sulfonated poly(arylene ether sulfone)s. Macromolecules 42, 1873-1880 (2009).

37 Bae, B., Miyatake, K. \& Watanabe, M. Synthesis and properties of sulfonated block copolymers having fluorenyl groups for fuel-cell applications. ACS Appl. Mater. Interfaces 1, 1279-1286 (2009).

38 Bae, B., Yoda, T., Miyatake, K., Uchida, H. \& Watanabe, M. Proton-conductive aromatic ionomers containing highly sulfonated blocks for high-temperature-operable fuel cells. Angew. Chem. Int. Ed. 49, 317-320 (2010).

39 Bae, B., Miyatake, K. \& Watanabe, M. Sulfonated poly(arylene ether sulfone ketone) multiblock copolymers with highly sulfonated block. Synthesis and properties. Macromolecules 43, 2684-2691 (2010).

40 Miyatake, K., Hirayama, D., Bae, B. \& Watanabe, M. Block poly(arylene ether sulfone ketone)s containing densely sulfonated linear hydrophilic segments as proton conductive membranes. Polym. Chem. 3, 2517-2522 (2012).

41 Miyake, J., Watanabe, M. \& Miyatake, K. Sulfonated poly(arylene ether phosphine oxide ketone) block copolymers as oxidatively stable proton conductive membranes. ACS Appl. Mater. Interfaces 5, 5903-5907 (2013).

42 Akiyama, R., Hirayama, D., Saito, M., Miyake, J., Watanabe, M. \& Miyatake, K. Proton conductive aromatic block copolymers from a new bistriazole monomer. RSC Adv. 3, 20202-20208 (2013) 